\title{
Finite element method application for simulation of ground penetrating radar response
}

\author{
M. Pasternak, R. Kędzierawski \& J. Pietrasiński \\ Military University of Technology, Warsaw, Poland
}

\begin{abstract}
The software for the simulation of electromagnetic waves propagation through the soil structure with buried objects has been described in the paper. The calculations are based on the finite element method (FEM) and allow prediction of the ground penetrating radar (GPR) echo structure from different buried objects. Such a virtual radar seems to be useful for test of chosen objects detection possibility in different soil structure configurations as well as for test of different kinds of radar signals. The comparison of simulation results and real measurements data has been also presented and discussed in the paper.
\end{abstract}

Keywords: finite element method (FEM), ground penetrating radar (GPR).

\section{Introduction}

The most popular method for simulation of electromagnetic wave propagation inside the soil structure is finite difference time domain (FDTD) method $[1,2]$. The main reason for the FDTD method popularity is its relative simplicity and good enough accuracy. An alternative approach is possible using finite element method (FEM). The solutions in this case are easier for interpretation and it gives more precise information about material discontinuities. The last feature is very important as far as ground penetrating radar working conditions are concerned. Additionally, the construction of the simulation boundaries in the FEM approach is easier and more precise [3]. Contrary to the FDTD in the case of FEM method the main field quantity characterizes all elements of volume instead of nodes. The material properties $\varepsilon, \mu$ and $\sigma$ are assumed to be constant inside the single simulation cells. 


\section{Simulation description}

To obtain the discrete centered differential equations spatial shift between magnetic and electric field components is assumed. The magnetic field components operate among simulation cells around $[i, j, k]$ node and occupy $1 / 8$ volume of elements $[i, j, k],[i+1, j, k],[i, j+1, k],[i, j, k+1],[i+1, j+1, k]$, $[i+1, j, k+1],[i, j+1, k+1]$ and $[i+1, j+1, k+1]$.

For time derivative centering the Yee method was used. The electric field is defined for $t=n \Delta t$ and magnetic one for $t=(n+1 / 2) \Delta t$.

For simulation the integral forms of Maxwell equations was used:

$$
\begin{gathered}
\oint \mathbf{E} \cdot \mathbf{d} \mathbf{l}=-\iint d A \mu \frac{\partial}{\partial t}(\mathbf{H} \cdot \mathbf{n}) \\
\oint \mathbf{H} \cdot \mathbf{d} \mathbf{l}=\iint d A\left[\varepsilon \frac{\partial}{\partial t}(\mathbf{E} \cdot \mathbf{n})+\mathbf{J}_{0}+\sigma \mathbf{E} \cdot \mathbf{n}\right]
\end{gathered}
$$

The contour integral of magnetic field on the left hand side of equation (2) is calculated with positive rotation along the path presented in the Fig 1.

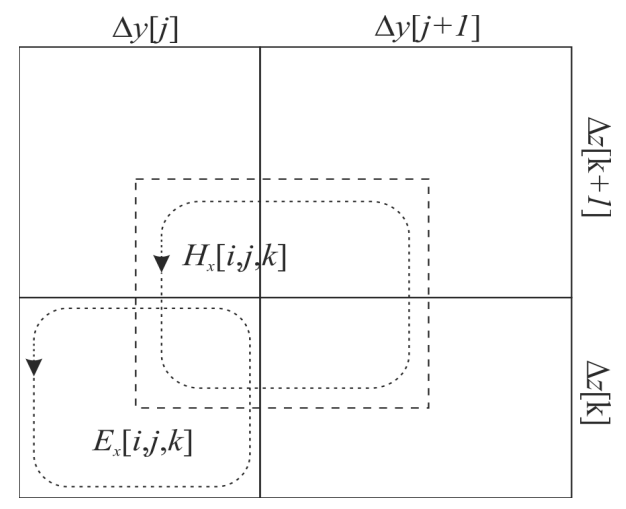

Figure 1: $\quad$ Magnetic and electric fields calculation path example [3].

The magnetic field component overlapping neighbour elements is averaged in $x$ direction. Summing up of all components the following integral formula around $[i, j, k]$ node can be obtained:

$$
\begin{aligned}
& \text { Int } H_{x}^{n+1 / 2}[i, j, k]=\left\{H_{y}^{n+1 / 2}[i-1, j-1, k-1]+H_{y}^{n+1 / 2}[i, j-1, k-1]+\right. \\
& +H_{y}^{n+1 / 2}[i-1, j, k-1]+H_{y}^{n+1 / 2}[i, j, k-1]-H_{y}^{n+1 / 2}[i,-1 j, k]+ \\
& \left.-H_{y}^{n+1 / 2}[i, j, k]-H_{y}^{n+1 / 2}[i-1, j-1, k]-H_{y}^{n+1 / 2}[i, j-1, k]\right\} \Delta y[j] / 4+ \\
& +\left\{H_{z}^{n+1 / 2}[i-1, j, k-1]+H_{z}^{n+1 / 2}[i, j, k-1]+H_{z}^{n+1 / 2}[i-1, j, k]+\right. \\
& +H_{z}^{n+1 / 2}[i, j, k]-H_{z}^{n+1 / 2}[i-1, j-1, k]-H_{z}^{n+1 / 2}[i, j-1, k]+ \\
& \left.-H_{z}^{n+1 / 2}[i-1, j-1, k-1]-H_{z}^{n+1 / 2}[i, j-1, k-1]\right\} \Delta z[k] / 4 .
\end{aligned}
$$


The field component of the surface integral on the right-hand side of (1) is equal to:

$$
\begin{aligned}
& \left\{\varepsilon[i, j, k] \frac{E_{x}^{n+1}[i, j, k]-E_{x}^{n}[i, j, k]}{\Delta t}+J_{0 x}^{n+\frac{1}{2}}+\right. \\
& \left.+\sigma[i, j, k] \frac{E_{x}^{n+1}[i, j, k]+E_{x}^{n}[i, j, k]}{2}\right\} \Delta y[j] \Delta z[k]
\end{aligned}
$$

In the form (4) the terms containing electric field are centered in time $t=(n+1 / 2) \Delta t$. From (3) and (4) the electric field component can be obtained in the following form:

$$
\begin{aligned}
& E_{x}^{n+1}[i, j, k]=\frac{1}{\frac{2 \varepsilon[i, j, k]}{\Delta t}+\sigma[i, j, k]}\left\{\left(\frac{2 \varepsilon[i, j, k]}{\Delta t}-\sigma[i, j, k]\right) E_{x}^{n}+\right. \\
& \left.-2 J_{0 x}^{n+1 / 2}+\frac{2 \operatorname{Int} H_{x}^{n+1 / 2}}{\Delta y[j] \Delta z[k]}\right\}
\end{aligned}
$$

The electric field can be calculated from (2). It has the form as follows:

$$
\begin{aligned}
& \text { Int } E_{x}^{n}[i, j, k]=\left\{E_{y}^{n}[i, j, k]+E_{y}^{n}[i+1, j, k]+E_{y}^{n}[i, j+1, k]+\right. \\
& +E_{y}^{n}[i+1, j+1, k]-E_{y}^{n}[i, j, k+1]-E_{y}^{n}[i+1, j, k+1]+ \\
& \left.-E_{y}^{n}[i, j+1, k+1]-E_{y}^{n}[i+1, j+1, k+1]\right\} \Delta y[j] / 4+ \\
& +\left\{E_{z}^{n}[i, j+1, k]+E_{z}^{n}[i+1, j+1, k]+E_{z}^{n}[i, j+1, k+1]+\right. \\
& +E_{z}^{n}[i+1, j+1, k+1]+E_{z}^{n}[i, j, k]-E_{z}^{n}[i+1, j, k]+ \\
& \left.-E_{z}^{n}[i, j, k+1]-E_{z}^{n}[i+1, j, k+1]\right\} \Delta z[k] / 4 .
\end{aligned}
$$

The surface integral from right-hand side of (2) contains the magnetic permittivity averaged from 8 neighbour cells. Using time centered derivative it has got the following form:

$$
\begin{aligned}
& \frac{1}{8}[(\mu[i, j, k]+\mu[i+1, j, k]) \Delta y[j] \Delta z[k]+ \\
& (\mu[i, j+1, k]+\mu[i+1, j+1, k]) \Delta y[j+1] \Delta z[k]+ \\
& (\mu[i, j, k+1]+\mu[i+1, j, k+1]) \Delta y[j] \Delta z[k+1]+ \\
& (\mu[i, j+1, k+1]+\mu[i+1, j+1, k+1]) \Delta y[j+1] \Delta z[k+1]] \times \\
& \frac{H_{x}^{n+\frac{1}{2}}[i, j, k]-H_{x}^{n-\frac{1}{2}}[i, j, k]}{\Delta t}
\end{aligned}
$$


Based on (6) and (7) the magnetic field components obtain the form as follows:

$$
H_{x}^{n+\frac{1}{2}}[i, j, k]=H_{x}^{n-\frac{1}{2}}[i, j, k]-\frac{\Delta t}{M u A_{x}} \operatorname{Int} E_{x}^{n}[i, j, k],
$$

where

$$
\begin{aligned}
& M u A_{x}=\frac{1}{8}[(\mu[i, j, k]+\mu[i+1, j, k]) \Delta y[j] \Delta z[k]+ \\
& (\mu[i, j+1, k]+\mu[i+1, j+1, k]) \Delta y[j+1] \Delta z[k]+ \\
& (\mu[i, j, k+1]+\mu[i+1, j, k+1]) \Delta y[j] \Delta z[k+1]+ \\
& (\mu[i, j+1, k+1]+\mu[i+1, j+1, k+1]) \Delta y[j+1] \Delta z[k+1]] .
\end{aligned}
$$

The remaining components of $\boldsymbol{E}$ and $\boldsymbol{H}$ were calculated in the same way.

In the first step of simulation the electric field components are actualized by directly replacing old values by new ones, because they are independent of neighbour values of the field. Next the magnetic field is actualized.

The time step of the method has to be shorter than minimum field propagation time through the smallest simulation element.

If $\mathrm{v}$ is the electromagnetic wave velocity inside the element the well-known from the FDTD method Courant criterion has the following form:

$$
\Delta t \leq \min \left(\frac{\Delta x[i]}{v[i]}, \frac{\Delta y[j]}{v[j]}, \frac{\Delta z[k]}{v[k]}\right) .
$$

For appropriate time step, centre frequency $f_{0}$ and excitation pulse bandwidth $f_{\mathrm{w}}$ of the discretized excitation wave has the form:

$$
u(n)=\exp \left(-\left(\left(n-t_{0}\right) / T\right)^{2}\right) \sin \left(2 \pi f_{0} n \Delta t\right)
$$

where $T=1 /\left(f_{w} \Delta t\right), t_{0}=5 T$.

In the FEM method the boundary conditions formulation is relatively simple. The simulated region is usually surrounded by one layer with suitable material parameters. Out of the layer the nodes having fixed magnetic field values (usually equal to zero) are placed.

The ideal absorber has to behave like an open circuit with current value equal to the current induced by the incident field. It can be obtained by appropriate selection of boundary layer specific conductivity.

When flat wave incidents perpendicularly to the boundary layer with specific conductivity $\sigma$ and thickness $\Delta$ and the layer has the same permittivities like neighbour material, $\Delta<<\lambda$ and electric component $E_{x}$ is constant in all layer 
thickness, then electric field induces current density $J_{x}=\sigma \Delta E_{x}$ in the layer. For $\boldsymbol{H}=0$ outside of the simulated space the magnetic field component $H_{y}$ inside is equal to $J_{x}$. Using well-known formula:

$$
\frac{E_{x}}{H_{y}}=\sqrt{\frac{\mu}{\varepsilon}}
$$

one can obtain the specific layer conductivity necessary to terminate the wave.

$$
\sigma=\frac{1}{\Delta \sqrt{\mu / \varepsilon}}
$$

If the incident angle is different from $90^{\circ}$ then effective thickness of the layer is equal to $\Delta / \sin \theta$.

\section{Model verification}

The model shortly described above was implemented to the software operating in Windows 7 x64 system and prepared using a six-core Pentium processor.

The software allows us to define up to ten soil layers with different thickness, permittivities and conductivities, as well as a random number of cuboidal objects. It is also possible to define the basic properties of antennas as well as radar signals. Thanks to the features mentioned above, the reconstruction of real conditions inside the simulated medium is possible with a fidelity good enough.

Significant increase of the layers or objects number makes the simulation duration time very long. The calculations concerning for example air and two soil layers with two buried objects takes about 14 hours in $0.7 \mathrm{~m}^{3}$ virtual sandbox.

Numerical calculations were verified in laboratory conditions using dry river sand and two buried objects: metal plate and wooden box as a surrogate of antipersonnel mine PMD - 7 (Fig. 2).
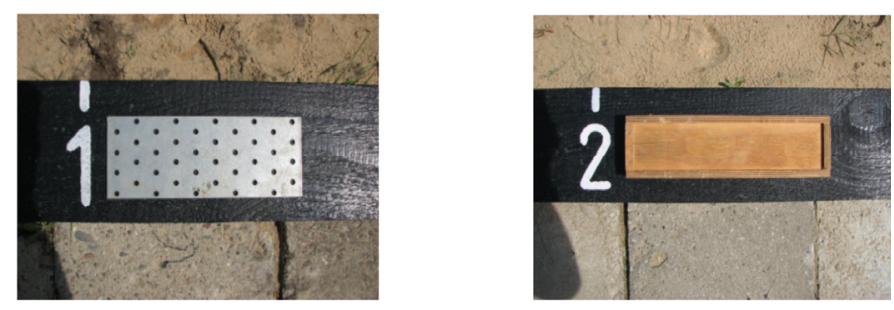

Figure 2: $\quad$ Test objects: 1 - metal plate, 2 - wooden box as an antipersonnel mine PMD-7 surrogate.

The measurements were carried out using vector network analyser and Vivaldi antennas inside anechoic chamber (Fig. 3). The stepped frequency continuous wave (SFCW) signal was generated in the range from about 1 to 3 
$\mathrm{GHz}$ and it consists of 512 steps with equal duration time and $4 \mathrm{MHz}$ single step. The results obtained in the frequency domain was recalculated into the time domain using inverse discrete Fourier transform with appropriate window function and zero -padding technique.

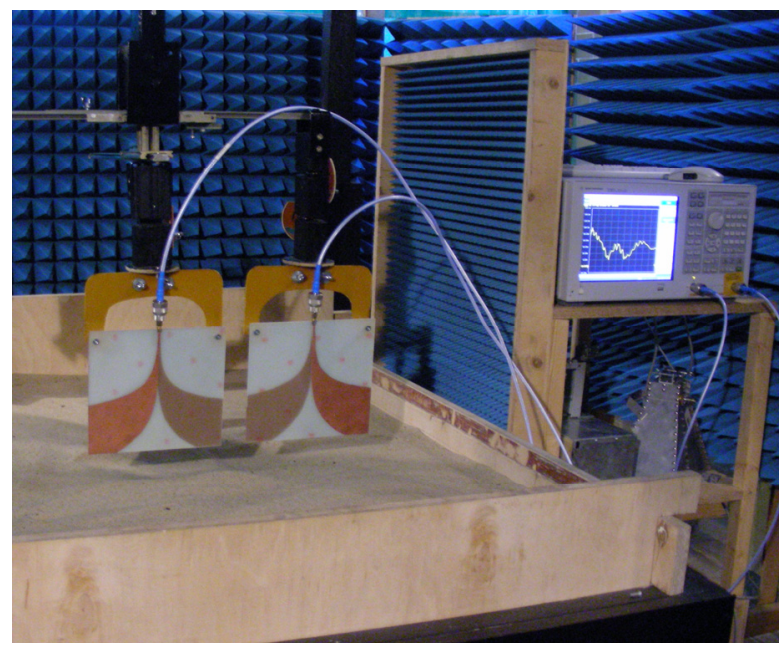

Figure 3: $\quad$ The measurement set.

The comparison of measurement and calculation of exemplary results for the objects shown in Fig. 2 is illustrated in Fig. 4.
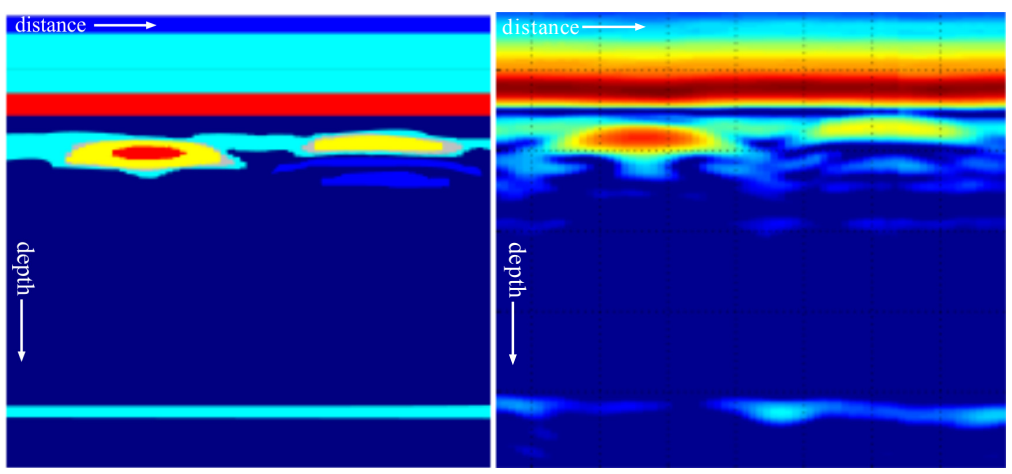

Figure 4: Comparison of calculation (on the left) and measurements (on the right).

The virtual and real environment conditions were similar as much as possible. The results both theoretical and measured data have very good similarity. Conclusions 
The FEM simulations of the GPR imaging seem to be more precise than in the FDTD case. Main reason of the difference is caused by numerical boundary conditions construction that is much easier for FEM method. The FDTD method models tested earlier by authors required much bigger simulated volume for absorbing boundary conditions implementation (PML) to obtain similar effects as FEM with one layer only.

The simulation results give very good similarity with real data from the laboratory environment. Moreover, it is possible to simulate GPR imaging in more realistic environment because the software is flexible enough. The simulation results analysis is very important from GPR image recognition problem point of view.

\section{Acknowledgement}

This work was supported by the Polish Ministry of Science and Higher Education from sources for science in the years 2009-2011 under project OR00006909.

\section{References}

[1] Taflove, A. \& Hagness, S. C., Computational electrodynamics the finitedifference time domain method, Artech House, 2000.

[2] Pasternak, M. \& Silko, D., Software for simulation of electromagnetic waves propagation through the soil with buried objects, Proc. of the $11^{\text {th }}$ International Radar Symposium, pp. 524-527, 2010.

[3] Humphries, S. Jr., Field Solutions on Computers, CRC Press, 1997. 